\title{
Application of Cryo-Electron Microscopy on Drug Discovery
}

Viswanath Vittaladevaram $^{1}$ and Kranthi Kuruti ${ }^{2}$

${ }^{1}$ BIODISCOVERY GROUP, Anantapur, India, ${ }^{2}$ Mahindra India, Tirupathi, India

\section{Abstract:}

The key aspect for development of novel drug molecules is to perform structural determination of target molecule associated with its ligand. One such tool that provides insights towards structure of molecule is Cryo-electron microscopy which covers biological targets that are intractable. Examination of proteins can be carried out in native state, as the samples are frozen at -175 degree Celsius i.e. cryogenic temperatures. In addition to this, there were no limits for molecular and functional structures of proteins that can be imagined in 3-dimensional form. This includes ligands which unravel mechanisms that are biologically relevant. This will enable to better understand the mechanisms that are used for development of new therapeutics. Application of Cryo-electron microscopy is not limited to protein complexes and is considered as non-specific. Intervention of Cryo-EM would allow to analyse the structures and also able to dissect the interaction with therapeutic molecules. The study determines the usage of cryo-EM for providing resolutions that are acceptable for lead discovery. It also provides support for lead optimization and also for discovery of vaccines and therapeutics.

\section{References}

Bartesaghi, A., Merk, A., Banerjee, S., Matthies, D., Wu, X., Milne, J. L., \& Subramaniam, S. (2015). 2.2 $\AA$ resolution cryo-EM structure of $\beta$-galactosidase in complex with a cell-permeant inhibitor. Science (New York, N.Y.), 348(6239), 1147-1151. https://doi.org/10.1126/science.aab1576

Bartesaghi, A., Aguerrebere, C., Falconieri, V., Banerjee, S., Earl, L. A., Zhu, X., Grigorieff, N., Milne, J., Sapiro, G., Wu, X., \& Subramaniam, S. (2018). Atomic Resolution Cryo-EM Structure of $\beta$ Galactosidase. Structure (London, England $\quad$ : $\quad$ 1993), 26(6), $\quad$ 848-856.e3. https://doi.org/10.1016/j.str.2018.04.004

Kühlbrandt W. (2014). Biochemistry. The resolution revolution. Science (New York, N.Y.), 343(6178), 1443-1444. https://doi.org/10.1126/science.1251652

Renaud, J. P., Chari, A., Ciferri, C., Liu, W. T., Rémigy, H. W., Stark, H., \&Wiesmann, C. (2018). CryoEM in drug discovery: achievements, limitations and prospects. Nature reviews. Drug discovery, 17(7), 471-492. https://doi.org/10.1038/nrd.2018.77

Scheres S. H. (2012). A Bayesian view on cryo-EM structure determination. Journal of molecular biology, 415(2), 406-418. https://doi.org/10.1016/j.jmb.2011.11.010 